\title{
Das Arztzeugnis im Brennpunkt unterschiedlicher Interessen
}

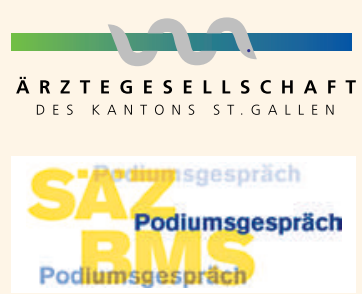

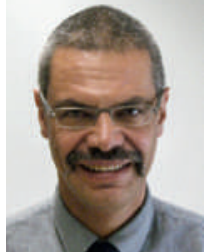

Andreas

Klipstein

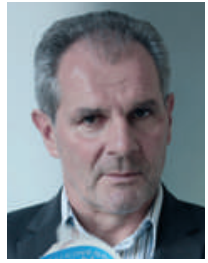

Albert

Koller

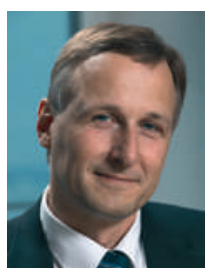

Roland Müller

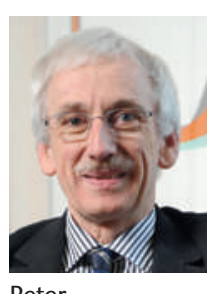

Peter

Wiedersheim

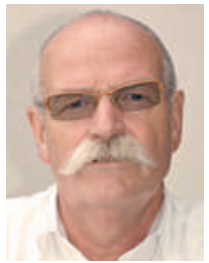

Markus

Löliger
Das Ausstellen von Zeugnissen, in denen die Arbeits(un)fähigkeit von Patienten festgehalten wird, gehört für Ärztinnen und Ärzte zum Berufsalltag. Obwohl es sich um einen «Routinevorgang» handelt, ist die Beurteilung der Arbeitsfähigkeit eine anspruchsvolle Aufgabe, bei der ein komplexes Zusammenspiel von Faktoren zu berücksichtigen ist. Ärztinnen und Ärzte bewegen sich dabei in einem Spannungsfeld von medizinischen Befunden sowie Erwartungen seitens der Patienten, der Arbeitgeber und im weiteren Sinne auch der Gesellschaft.

Die Ärztegesellschaft des Kantons St. Gallen hat in Sachen Arbeitszeugnis neue Wege beschritten und auch den Kontakt zur Arbeitgeberseite gesucht. Entstanden ist ein pragmatisches Modell mit einem einfachen und einem detaillierten Zeugnis, die sich in der Praxis gut bewähren.

\section{Diskutieren Sie mit}

Wo liegen die besonderen Anforderungen an Ärztinnen und Ärzte beim Ausstellen von Arbeitszeugnissen? Wie gehen sie sinnvollerweise damit um? Wie kann dem Patientenwohl oberste Priorität eingeräumt und gleichzeitig der Gefahr von «Gefälligkeitszeugnissen» begegnet werden? Welche juristischen Rahmenbedingungen und Stolpersteine sind zu beachten? Wie kann konstruktiv mit der Arbeitgeberseite zusammengearbeitet werden, ohne die Interessen des Patienten zu verletzen?

Mit dem Podium in St. Gallen möchte die Schweizerische Ärztezeitung zu einer fundierten Auseinandersetzung mit diesen und weiteren Fragen rund um

\section{Podiumsgäste}

Auf dem Podium diskutieren unter der Leitung von Markus Löliger, Journalist und Medienbeauftragter der Ärztegesellschaft des Kantons St. Gallen:

- PD Dr. med. Andreas Klipstein, Präsident Swiss Insurance Medicine (SIM)

- Albert Koller, Leiter Personalmanagement \& Academy der Jansen AG, Oberriet SC das Thema des ärztlichen Arbeitszeugnisses beitragen. Der Einbezug des Publikums in die Diskussion ist zentraler Bestandteil des Konzepts der SÄZ-Podiumsveranstaltungen.

\section{Datum / Zeit / Ort}

Die Podiumsveranstaltung mit anschliessendem Apéro findet statt am Donnerstag, 5. Dezember 2013, 19.00-21.00 Uhr, im Hotel Radisson Blu, St.Jakob Strasse 55, St. Gallen.

\section{Anmeldung}

Der Eintritt zur Veranstaltung ist frei, eine Anmeldung ist aber erforderlich. Anmeldungen können bis Dienstag, 3. Dezember 2013 vorgenommen werden, via E-Mail an redaktion.saez[at]emh.ch oder via Fax an 06146785 56. Bitte Ihren Namen und die Namen allfälliger Begleitpersonen sowie das Stichwort «Anmeldung zum SÄZ-Podium vom 5. Dezember» angeben. Auch telefonische Anmeldungen sind vormittags unter 0614678572 möglich.

\section{Veranstaltungspartner}

Die Podiumsdiskussion wird in Zusammenarbeit mit der Ärztegesellschaft des Kantons St. Gallen organisiert. Die Durchführung des Anlasses wird möglich dank grosszügiger Unterstützung durch Interpharma, den Verband der forschenden pharmazeutischen Industrie. Die Verantwortung für Konzept und Inhalt des Podiums liegt bei der Schweizerischen Ärztezeitung und der Ärztegesellschaft des Kantons St. Gallen.

- Prof. Dr. iur. Roland Müller, Rechtsanwalt und Lehrbeauftragter für Arbeitsrecht an den Universitäten St.Gallen und Bern

- Dr. med. Peter Wiedersheim, Präsident der Ärztegesellschaft des Kantons St. Gallen, Facharzt für Innere Medizin und Rheumatologie 